\title{
A FIBRE-OPTIC-TO-PNEUMATIC REMOTE FLOW CONTROLLER
}

\author{
John R. TIPPETTS \\ Department of Mechanical and Process Engineering \\ University of Sheffield \\ Mappin Street \\ Sheffield S1 4DU \\ England
}

\begin{abstract}
A laboratory demonstration system has been made which enables a signal carried by an optical fibre to control the operation of either a pneumatic actuator or a fluidic flow control valve. Apart from the actuator, all functions are implemented by no-moving-part fluidic amplifiers. Notably this includes the direct fluidic detection of the signal which is done using a commercially available sub-unit originally developed in the USA for defence applications. The system demonstrates the integration of signal-fluidics and power fluidics and shows how remote control can be implemented without the use of electrical signalling or power at the site of the actuator. Signals are produced by a laser diode. Control functions are the operation of a standard $100 \mathrm{~mm}$ bore butterfly valve or the 4-to-1 modulation or diversion of airflow by a switched vortex valve.
\end{abstract}

\section{KEY WORDS}

optical, fibre, fluidic, remote, control

\section{INTRODUCTION}

The work described here is motivated by the need for improved options for remotely controlling processing plant. With the increasing use of computer control there is an increasing need to deliver the control signals reliably and quickly to remote actuators or subsystems. The distances over which such signals may be

\begin{abstract}
sent, and the frequency of modulation are both also increasing. Traditional methods such as wire-born electrical transmission or pneumatic signalling have significant drawbacks which include vulnerability to electromagnetic interference and limited bandwidth. At the receiving end, if mechanical power is needed, the source of power is frequently electrical. Another demand for electrical power may be electronic signal
\end{abstract}


detectors and amplifiers. This requires more wiring, local generation or battery storage of electrical energy. The vulnerability and expense of such electrically operated systems can be avoided if pneumatic (or gaspressure) power is already installed. The objective of the system described here is to take advantage of such sources of fluid power thereby avoiding the need for electrical supplies. A further objective is to minimise the number of moving-part components so as to avoid wear-induced failure. Only one moving-part component is used, and this is a well-proven quarterturn valve actuator and butterfly valve .

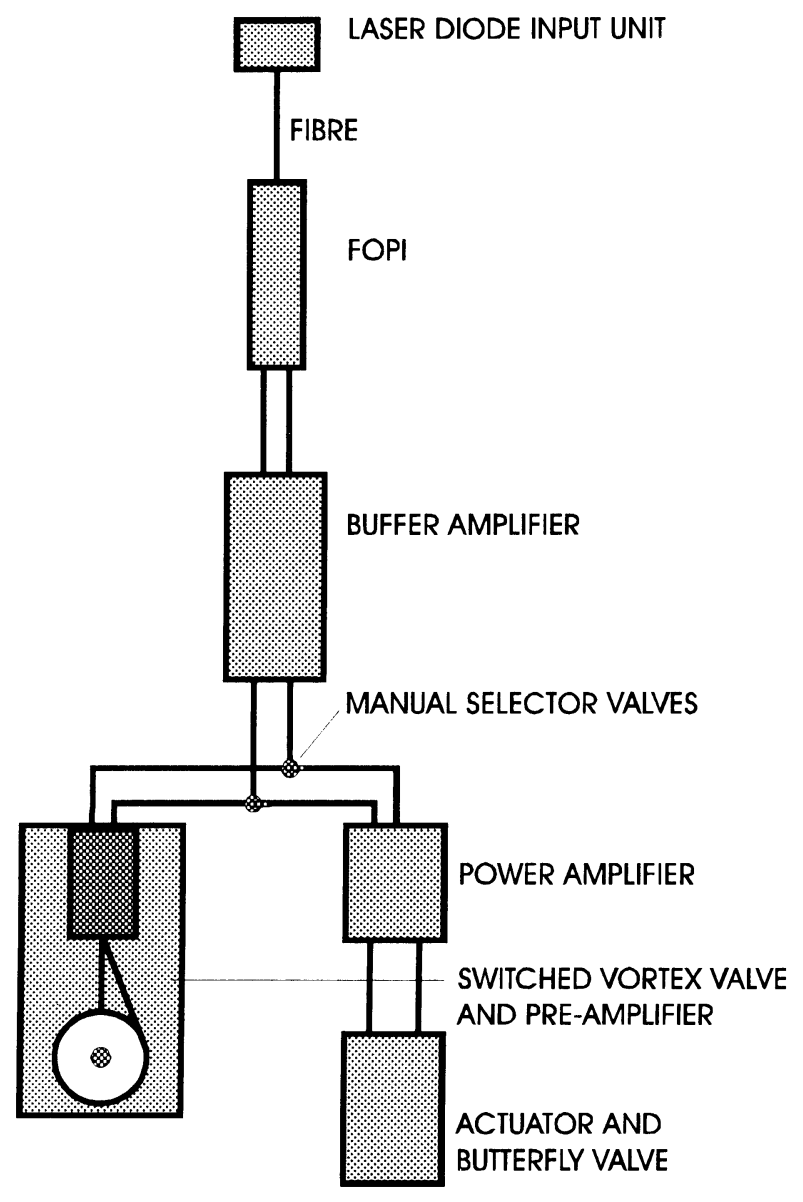

Fig 1 System components

\section{OBJECTIVES}

A wide audience was aimed at: For those unfamiliar with fluidics, the system should show the scope of the technology: i.e the detection of almost imperceptible signals, signal processing, power amplification and flow modulation. For those more familiar with fluidics it should demonstrate the unification of microscopic signal-processing and "heavy-current-" or powercontrol and the further integration of carefully chosen conventional moving-part technology. It should also enable process- and control-engineers to envisage future use of the techniques of pure fluid power control and its direct interface with state-of-the-art signal transmission methods, in particular optically based modes. In other words, to dissuade them from thinking only of electronic and electrical signal reception and amplification.

Detailed Specification

The detailed specification follows from the overall block diagram as shown in Fig 1. This comprises 1) an electronic signal sending unit enabling an on-off switch to put appropriate laser signals into an optical fibre.

2) a pneumatic manual input method using a simple on-off valve to send a small pressure signal into $3 \mathrm{~mm}$ bore tubes.

3) a fluidic fibre-optic-to-pneumatic interface detector and amplifier ("FOPI") to receive and amplify the signals from the optical fibre.

4) a series of vented fluidic amplifiers able primarily to amplify pressure signals to a sufficient level for power control.

5) a series of unvented fluid amplifiers able primarily to amplify flow signals.

6) a fluidic switched vortex device to demonstrate direct fluidic flow control, either turning down the main flow or diverting it.

7) the components of the demonstration system should easily come apart for observation as self-contained subsystems.

8) the whole system should be demonstrable without unusual safety or power-supply demands. Pneumatics rather than hydraulics was chosen to simplify the containment and demountability of the system. 9) two modes of operation should demonstrate:a) useful operation at the lowest possible supply pressure, and b) high power operation when the supply pressure is not limited.

\section{SYSTEM COMPONENTS}

Fibre-optic-to-pneumatic interface (FOPI) 


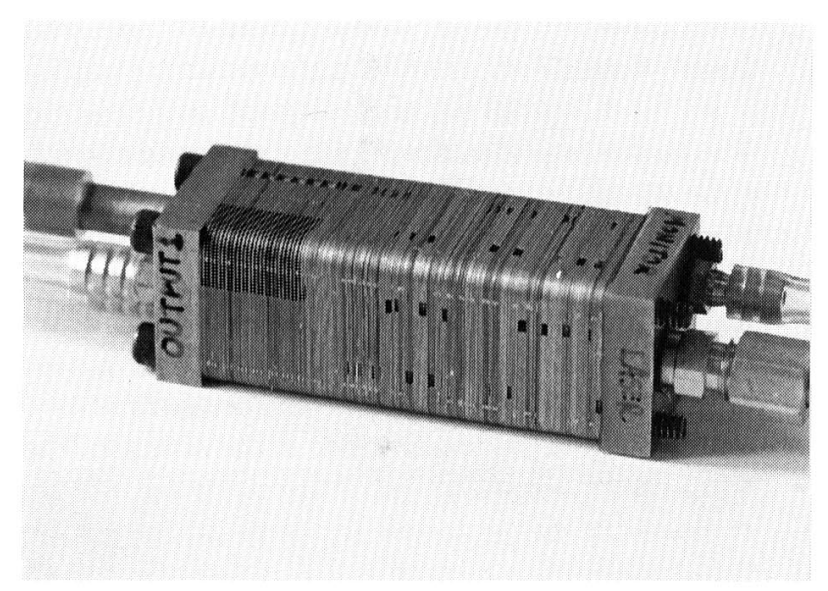

Fig 2 Fibre-optic-to pneumatic interface.

The interface between the laser control signal and the fluid power medium is embodied in a special subcircuit type FOPI-1010/130 obtained from DRT, Maryland USA. The unit shown in fig 2 is about 75 $\mathrm{mm}$ long. The fibre enters at the right. On the left are the output tubes and the air supply. Vented flow emerges from the edges of the stack. As described by Drzewiecki in [1], the heart of the FOPI is a laminarflow fluid amplifier in which the normal pair of control ports has been replaced by a single control channel with a dead end forming a small cavity. A window in the cover plate of the amplifier allows light or infra-red signals to heat the inside of the cavity thereby producing control flows by the thermo-acoustic effect. This signal is then amplified by the same process as in a normal laminar fluidic element. Several stages of amplification then produce a usefully large signal. The necessary multi-stacking and circuit construction is a special feature of these micro-fluidic components so the whole module becomes a single block with one pneumatic supply port, two output ports, and numerous vents to atmosphere.

The nominal signal is $5 \mathrm{~mW}$ of infra-red radiation modulated at $3500 \mathrm{~Hz}$. After amplification and demodulation via a high-pass filter this gives a steady signal in one of the output ports. If the modulation falls outside the range 1500 to $5000 \mathrm{~Hz}$ the fluid dynamic signal is too weak to be detected and the logical level of the input is then zero. Much more selectivity can be incorporated to allow more control options but this was unnecessary in the demonstration system. The input signal is provided by a laser diode having an output of about $20 \mathrm{~mW}$ at a wavelength of $786 \mathrm{~nm}$. The air supply pressure and flow to the FOPI are nominally 0.17 barg and 7.5 SLM (standard litres $/ \mathrm{min}$ ). The output can give a flow of 2 SLM at 0.0533 bar differential pressure. Transmission distances of 400 metres have been routinely used by DRT (in the US). In theory many kilometres should be possible.

\section{Buffer Amplifier}

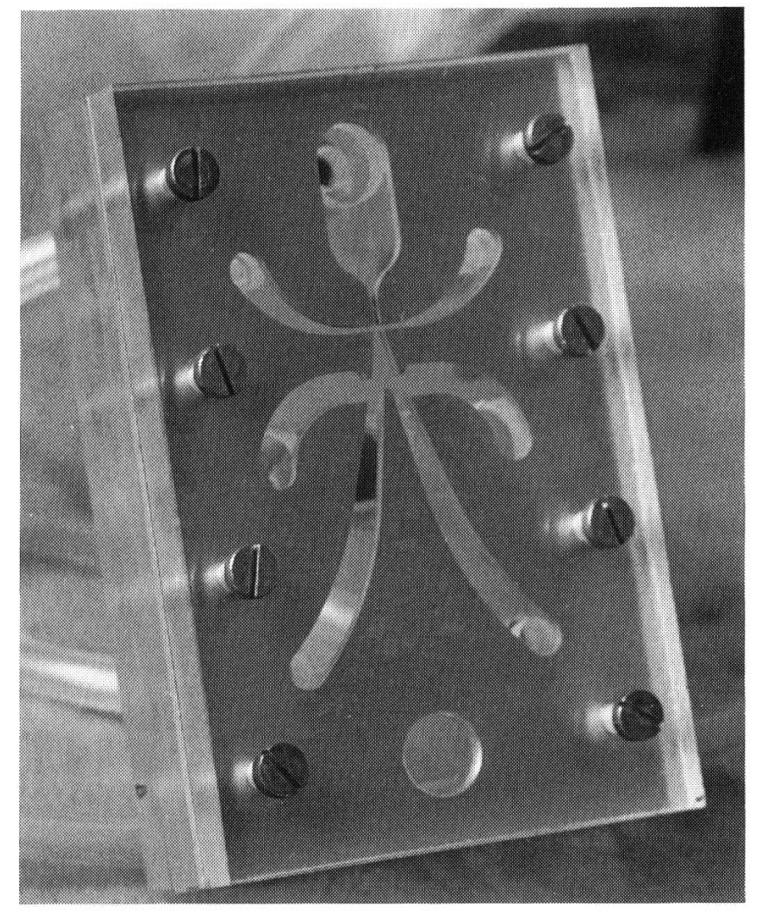

Fig 3 Vented fluidic amplifier.

(wire-cut from duralumin $60 \times 80 \mathrm{~mm}, 2 \mathrm{~mm}$ thick) This is actually the power amplifier but it is identical to those in the buffer amplifier.

The signal from the FOPI is amplified by a buffer amplifier module comprising two vented wallattachment fluid amplifiers operating with turbulent flow. The significance of this is that there is no strict upper limit to the Reynolds number so the only limitation on flows and pressures is due to compressibility effects. The two amplifiers (of the type shown in fig 3) are identical and are very similar to standard fluid logic flip-flops, however their size is larger, having a channel depth of $2 \mathrm{~mm}$ and a main nozzle width of $1 \mathrm{~mm}$. The signal is boosted by carefully matching the supply pressures to the two 
stages in relation to each other and to the output from the FOPI. The choice of pressures also depends on the desired function to be demonstrated: Maximum pressure gain corresponds to demonstrating a high pneumatic output power for fast actuation of valves or cylinders. An intermediate level of gain corresponds to demonstrating a system that can operate with the smallest possible overall supply pressure (or standby flow usage). Yet another gain level may be chosen to operate the fluidic flow control valve.

The output available from the amplifier is a pressureflow output characteristic measured at the active ("ON-side") output port. The other ("passive" or "OFFside") port is essentially at atmospheric pressure and is virtually completely decoupled from the active port. Although comprehensive characteristics are complicated a useful rule in quantifying the available output flow and pressure is:

output $=($ supply flow $) / 3$ at $($ supply pressure $) / 4$

The effect on the input characteristics is more complicated because they tend to be more sensitive to both Reynolds- and Mach number. The dual control port input also complicates simple quantification of "gain" or "amplification". Despite this, for the purposes of explanation to those unfamiliar with fluid amplifiers we can enunciate another simple rule: that switching can be initiated by a differential control pressure and flow given by

control $=($ supply flow $) / 50$ at $($ supply pressure $) / 25$

Combining the two rules above shows that the order of magnitude of the gain available per amplifier can be judged on the basis of about 6.25 for pressure and about 16 for flow. Numerous factors can influence these values however. To some extent flow and pressure gain can be traded off but flow gain is easier to achieve than pressure gain.

The pressure gain figure suggests that the supply pressure of the second stage should be set at 6.25 times that of the first stage. In fact, to give a safety margin for reliable operation over a wide range of supply pressures, the ratio was set at 4.6. This was built-in by a fixed orifice-type restrictor in the supply pipework so the buffer amplifier module is supplied by a single connection.
Power Amplifier

The power amplifier driving the valve actuator is simply another vented wall-attachment amplifier identical to the two used for the buffer module. In this application an important feature of the amplifier is its ability to drive a double-acting type load. This means that when supplying flow through the active outlet, the passive outlet must accept an equal return flow displaced from the inactive side of the actuator. The amplifier is natuarally able to accommodate this reverse flow, allowing it to escape to atmosphere via the vent.

\section{Unvented Preamplifier}

The second channel of signal-amplification leads to the fluidic switched-vortex valve. The requirement here is primarily flow amplification because the objective is that the fluidic valve exerts its control influence on large volumes of low pressure air, as in a ventilation system or combustion-air control application. For this purpose a series of unvented wall-attachment fluid amplifiers is connected to amplify the very small flow signals from the buffer amplifier (or, in fact, directly from the FOPI-unit) and inject them into the switched vortex device. The basic amplifier profile is replicated to form a 3-stage module able to accept signals from the buffer amplifier or the FOPI unit to switch the high-flow switched-vortex valve. Starting with a device having a $1 \times 4 \mathrm{~mm}$ supply nozzle, the crosssectional supply nozzle areas are increased in successive stages by a factor of about 6 . For convenience of construction, the aspect ratio (depth/width) of each amplifier can be fairly freely chosen to give neat packaging. Consequently, the third-stage amplifier has the unusually large aspect ratio of 7.6, but this enables it to fit neatly onto the switched vortex device. The rational design of such amplifying chains has been described in [2]

\section{Switched Vortex Valve}

The switched vortex valve ("SVV", variously named, sometimes as a "coanda switched vortex valve") is the combination of a fluidic diverter and a vortex valve as invented by Adams and Moore [3], its optimisation and other features have been described in [ $4 \& 5]$. In this application it is used to control low pressure air 
flow either throttling it or diverting it by using two different outlet configurations of the valve. In one configuration the outlet is in the form of a conical diffuser which suits the flow-turndown mode of operation whereby the throughflow is either maximum or reduced to $25 \%$ of its value by switching the valve from the non-vortex to the vortex state. Using another outlet configuration, a nozzle and plenum-mountedreceiver, the flow is reduced and diverted into a second outlet channel (connected to the plenum).

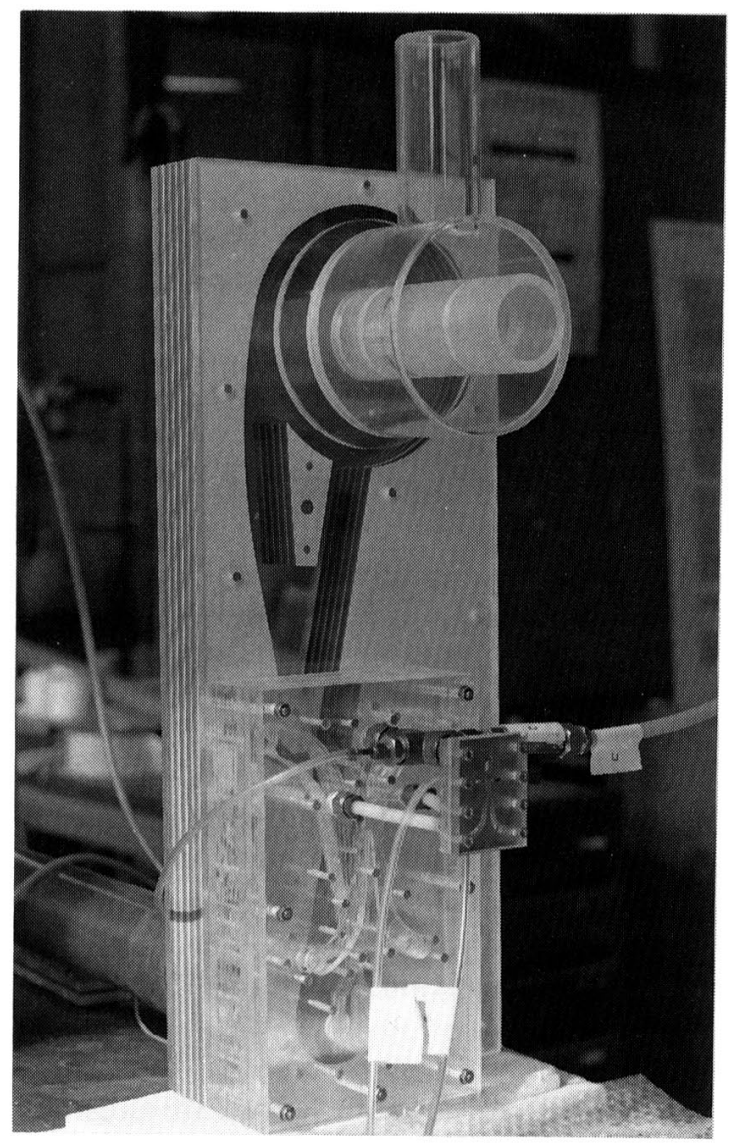

Fig 4 Switched vortex valve and pre-amplifier.

The SVV is laser cut from laminations of plastic foamboard clamped between acrylic plates. Most of the other components are acrylic. The pre-amplifier is a block of two closely integrated amplifiers built onto the front plate of the switched vortex valve and a pipeconnected first-stage amplifier (closest to camera). The plenum-mounted diverter is shown attached to the SVV outlet. Non-vortex flow emerges axially from the diffuser facing the camera, and vortex flow, captured by the plenum discharges from the vertical pipe-stub.

The SVV has a $19 \times 50 \mathrm{~mm}$ supply nozzle and carries a nominal maximum air flow of $1.5 \mathrm{cu}$ metre/min supplied in the demonstration set-up by a fan having a nominal output pressure of 0.017 barg.

Pneumatically Actuated Butterfly Valve

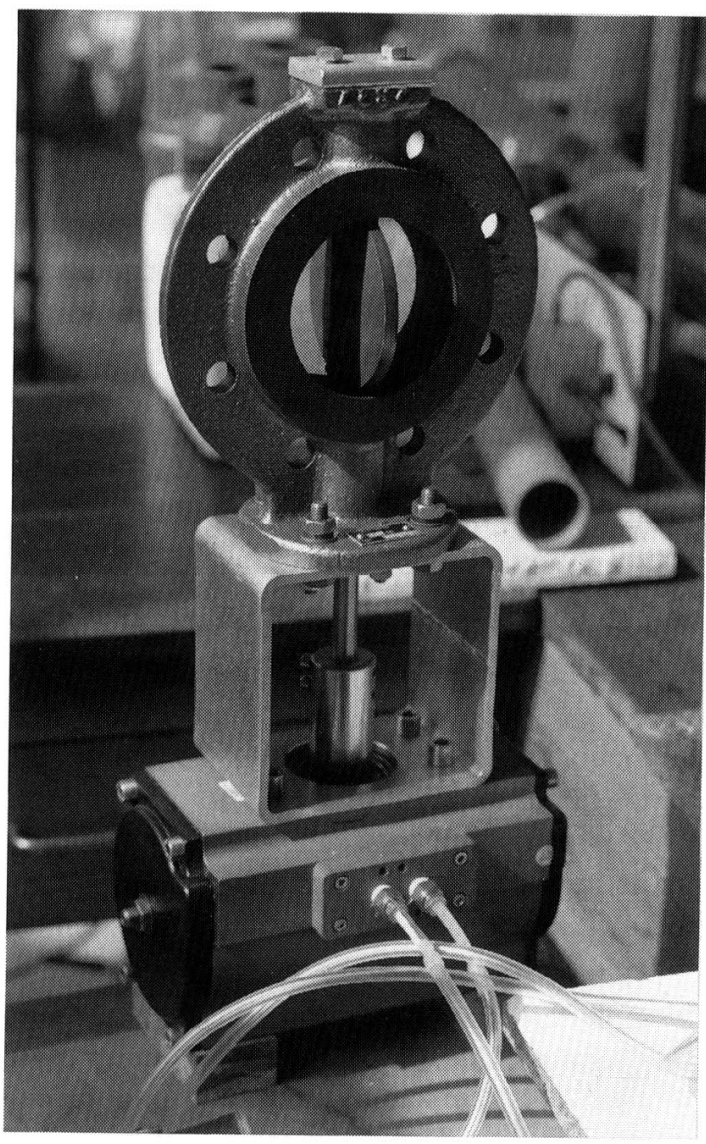

Fig 5 Pneumatically actuated butterfly valve

This unit comprises a Bettis-RP2253 double-acting pneumatic quarter-turn valve actuator, giving a torque of 46 newton-metres per bar pressure difference, and a $100 \mathrm{~mm}$-bore butterfly valve (from the Batley Valve Company) chosen to demonstrate a well-proven conventional valve integrated with fluidic control. The actuator and valve needed a port-pressure-difference of 0.03 bar to overcome friction so the fluidic system was 
easily able to operate it, providing a maximum available output differential pressure of 1.25 bar.

\section{OPERATING DATA}

Several modes of operation can be demonstrated:

1) Normal Operation of Butterfly Valve

The power amplifier is supplied at between 1 and 3 barg, while the FOPI and buffer amplifier are supplied at 0.15 and between 0.4 and 0.7 barg. Movement of the valve occurs within 1 second at the highest pressure settings.

\section{2) Butterfly Actuation with Minimum Air Usage}

Here the FOPI and the buffer amplifier alone are used to control the valve actuator (no power amplifier). Satisfactory operation occurs when the supply pressures to the FOPI and buffer amplifier are 0.15 and 0.2 barg respectively. The air flow usage is about 40 standard litres $/ \mathrm{min}$. As expected, the valve action is slow and takes about seven seconds to open or close. Even lower pressures can be used but this reduces the margin for contingencies.

3) Normal operation of the Switched Vortex Valve
Signals from the buffer amplifier are fed to the unvented pre-amplifier unit supplied at 0.09 bar which then controls the SVV supplied at 0.017 barg switching about $0.025 \mathrm{~m}^{3} / \mathrm{s}$ of ambient air.

4) SVV Minimal System

The FOPI will directly control the SVV by way of the pre-amplifier without the need for the buffer amplifier. With the FOPI supplied at 0.15 barg, operation is satisfactory up to a pre-amplifier supply pressure of $0.0115 \mathrm{barg}$, implying an SVV main pressure up to 0.023 barg

\section{CONCLUSION}

The system demonstrates the ability to combine "micro" and "macro" fluidics with a conventional valve controller and to implement remote control using optical fibres for transmission without the need for electronics at the receiving end. It is expected that systems with specific functions based on these techniques will be developed in the near future.

Acknowledgements

Contributors to this work were Professor R F Boucher, Drs S B M Beck, H Haider and G H Priestman.

\section{REFERENCES}

1 Drzewiecki T M, Holmes A B, Packard E A and Kiernan R J: Fluidic guidance for space-based kinetic energy weapon projectiles, Proc. Symp "FLUCOME", ASME ,Aug 1991 pp 303-311

2 Tippetts J R and Royle J K: Design of flow control circuits involving unvented bistable fluid amplifiers, Fluidics Quarterly, (issue 13) Vol 3 no 4 Oct 1971, pp1-15

3 Adams R B and Moore C B: US Patent 3,267,946, dated Aug 231966.

4 Syred N, Royle J K and Tippetts J R: Optimisation of High Gain Vortex Devices, Paper J3, proc 3rd Cranfield Fluidics Conference, BHRA, Bedford 1968

5 Goh Y R, Tippetts J R and Priestman G H: Optimisation of a Coanda Switched Vortex Valve, Proc FLUCOME symp. Vol 2, CERT-ONERA Toulouse 31055, (ISBN 2-84088-010-5) Aug 1994, pp 751-756 